\title{
DESCONHECIMENTO DA DIRETRIZ DE PREVENÇÃO DA ATEROSCLEROSE NA INFÂNCIA E ADOLESCÊNCIA POR PEDIATRAS EM SÃO PAULO
}

Adriana Ferreira Grosso ${ }^{1 *}$, Raul D. Santos ${ }^{2}$, Protásio L. da Luz ${ }^{3}$

Trabalho realizado no InCor HCFMUSP - Unidade Clínica de Aterosclerose, São Paulo, SP

*Correspondência: InCor HCFMUSP - Unidade Clínica de Aterosclerose Avenida Dr. Enéas de Carvalho Aguiar, 44 São Paulo - SP CEP: 05403-000

\section{RESUMO \\ OBjetivo. Relatar a extensão do conhecimento de pediatras de São Paulo sobre a diretriz.}

Métodos. Durante eventos de pediatria ou nos locais de trabalho, pediatras responderam um questionário contendo informações sobre o tempo de formação, local de trabalho, dedicação dentro da área de pediatria, atuação em área acadêmica e questões de múltipla escolha com informações retiradas da diretriz. As diferenças de expressão e associações foram consideradas significantes estatisticamente quando $p<0,05$. Todas as análises foram realizadas usando teste de Quiquadrado e teste de Quiquadrado de tendência linear por meio dos Softwares Primer of Biostatistics ou SPSS for Windows.

Resultados. Dos 370 pediatras entrevistados, 65,7\% afirmaram não ter tido conhecimento prévio da diretriz. O valor de corte para os acertos foi maior ou igual a $70 \%$ (maior ou igual a cinco questões corretas). Apenas 136 pediatras $(36,7 \%)$ alcançaram tal valor e dentre esses não havia relação no conhecimento da diretriz quanto aos sexos $(p=0,25)$. Dos 187 profissionais envolvidos em atividades acadêmicas, 45 (24\%) acertaram o valor de corte e dos 183 não participantes de atividades acadêmicas, $23(12,7 \%)$ acertaram maior ou igual a cinco questões $(p<0,001)$. Pediatras do setor público apresentaram melhor conhecimento da prática preventiva da aterosclerose $(46,1 \% ; p=0,01)$. 0 desconhecimento da diretriz foi independente do tempo de formação.

Conclusão. A meta da diretriz de servir como referência para o estabelecimento de estratégias individuais e populacionais no controle dos fatores de risco para a aterosclerose desde a infância não foi alcançada na cidade de São Paulo. A divulgação mais adequada e cursos de educação médica continuada em que o conhecimento da diretriz se torne mais efetivo são sugeridos para corrigir tais achados.

Unitermos: Prevenção primária. Aterosclerose. Criança. Adolescente. Diretrizes para o planejamento em saúde.

\section{INTRODUÇÃO}

As evidências atuais indicam que a aterosclerose se inicia na infância como um processo de agressão persistente ${ }^{1,2}$. Fatores de risco estão relacionados ao desenvolvimento de lesões ateroscleróticas desde os seus estágios mais precoces.

$O$ interesse pela aterosclerose na infância e na adolescência data do século XIX e, desde então, vêm sendo apresentados na literatura relatos da existência de lesões arteriais nessa faixa etária baseados em achados anatomopatológicos ${ }^{3,4}$.

O Bogalusa Heart Study ${ }^{5,6,7}$ analisou material de necrópsia e a presença antemorte de níveis séricos de lipoproteínas em diferentes grupos etários com início ao nascimento. A presença de placas fibrosas nas coronárias se associou a níveis elevados de triglicérides e pressão arterial. A extensão das estrias gordurosas na aorta estaria interligada diretamente aos níveis séricos elevados de colesterol total (CT) e lipoproteínas de baixa densidade (LDL) e inversamente interligada aos níveis de lipoproteínas de alta densidade (HDL). Tracy et al. ${ }^{7}$ mostraram que a presença de múltiplos fatores de risco concomitantes como altos níveis de CT, LDL e VLDL (lipoproteínas de muito baixa densidade) e hipertensão arterial (HA) associados as já comprovadas influências diretamente proporcionais dos níveis séricos do CT e das LDL e indiretamente das HDL na aorta, antes da morte, propiciaram o aparecimento de estrias gordurosas e agravaram a patogenia aterosclerótica nas coronárias e aorta, fortalecendo a ideia de sinergismo de efeito sobre a evolução das lesões ${ }^{5}$.

No estudo The Pathobiological Determinants of Atherosclerosis in Youth (PDAY) ${ }^{8,9}$, em cerca de 1530 necrópsias em indivíduos jovens entre 15 e 19 anos de idade, em metade das

1. Doutoranda pelo InCor Faculdade de Medicina da Universidade de São Paulo - FMUSP e docente titular do departamento de Ciências da Saúde UNINOVE, São Paulo, SP

2. Professor livre-docente pela Faculdade de Medicina da Universidade de São Paulo - FMUSP e diretor da unidade clínica de Lípides InCor Hospital das Clínicas da Faculdade de Medicina da Universidade de São Paulo, HCFMUSP, São Paulo, SP

3. Professor associado livre-docente pela Faculdade de Medicina da Universidade de São Paulo - FMUSP e diretor da unidade clínica de Aterosclerose InCor Hospital das Clínicas da Faculdade de Medicina da Universidade de São Paulo - HCFMUSP, São Paulo, SP 
coronárias e em todas as aortas havia lesões ateroscleróticas ${ }^{8}$. Há influência dos níveis de LDL e VLDL, intolerância à glicose, hipertensão arterial, tabagismo e obesidade na presença de níveis reduzidos de HDL na evolução precoce da aterosclerose em aorta e coronária direita quando da análise da progressão de lesões ateroscleróticas em jovens na faixa etária de 15 a 34 anos. Aproximadamente 3000 indivíduos mortos por causas externas tiveram amostras de sangue pós-mortem analisadas e concluiu-se que a prevalência e a extensão das lesões aumentaram rapidamente entre 15 e 34 anos de idade e que a doença aterosclerótica deve ser prevenida o mais precocemente possível ${ }^{9}$.

No Muscatine Study, o ultrassom de carótida foi utilizado para avaliação da espessura íntima-média das paredes vasculares e os dados foram associados aos níveis de CT e Índice de Massa Corporal $(\mathrm{IMC})^{10}$. Semelhantes resultados com carótidas foram obtidos no Bogalusa Heart Study ${ }^{11}$ e no Young Finns Study, reafirmando a ligação entre exposição a fatores de risco (LDL, IMC, pressão sistólica elevados e presença de tabagismo) em jovens e o aparecimento de aterosclerose subclínica no adulto jovem ${ }^{12}$.

Em relação aos estudos epidemiológicos no Brasil, Giuliano ${ }^{13}$ acompanhou 1053 indivíduos jovens do município de Florianópolis (SC) e constatou que níveis séricos elevados de colesterol acompanhavam o excesso de peso. Na região metropolitana do Rio de Janeiro (RJ), 68,4\% das crianças apresentaram alterações dislipidêmicas na faixa etária dos 5 aos 9 anos $^{14}$. Pellanda ${ }^{15}$, em 2002, enfatizou que aproximadamente $23 \%$ das crianças entre 6 e 12 anos e $21 \%$ entre 12 e 17 anos são obesas e que esta condição geralmente vem associada à hipertensão e ao diabetes. Um estudo transversal desenvolvido com aproximadamente 340 crianças e adolescentes entre 6 a 18 anos de idade na cidade de Fortaleza (CE) mostrou que $16,8 \%$ apresentaram sobrepeso e obesidade com pressão arterial acima do percentil 90 (44,7\%). Do total estudado, $51,5 \%, 38 \%$ e $15,5 \%$ dos avaliados eram, respectivamente, sedentários, tabagistas e etilistas.

A primeira diretriz de Prevenção da Aterosclerose na Infância e Adolescência ${ }^{16}$ foi lançada no ano de 2005 e, desde então, tornou-se referência para o estabelecimento de estratégias individuais e populacionais no controle dos fatores de risco para a prevenção precoce da aterosclerose. 0 objetivo deste estudo foi relatar a extensão do conhecimento de pediatras que atuam na cidade de São Paulo sobre a primeira diretriz de Prevenção da Aterosclerose na Infância e Adolescência. Uma vez que este profissional geralmente é o único que acompanha o indivíduo desde o nascimento e pode exercer fundamental papel na promoção da saúde cardiovascular.

\section{Métodos}

Foi utilizado o cálculo de amostra para estudo de prevalência transversal com populações finitas, chegando a uma amostra de 354 entrevistados. 0 percentual estimado conservador foi de $50 \%$ e erro amostral de $5 \%$.

Durante eventos de pediatria ou nos locais de trabalho, os pediatras foram convidados aleatoriamente a participar de uma pesquisa sobre conhecimento da diretriz. Os pesquisadores desconheciam a especialidade dos mesmos dentro da pediatria. O questionário continha informações sobre o tempo de formação, local de trabalho (redes pública e/ou particular), dedicação dentro da área de pediatria, atuação em área acadêmica e sete questões de múltipla escolha com informações retiradas da referida diretriz, durante o período de junho de 2008 a abril de 2009. Este estudo teve a aprovação da Comissão de Ética para Análise de Projetos de Pesquisa do HCFMUSP (CAPPesq).

As questões específicas e as respostas segundo recomendações da diretriz estão apresentadas na Tabela 1.

Ao entregarem as questões respondidas com o termo de consentimento livre e esclarecido, todos recebiam um folder contendo as respostas e a referência do site onde a diretriz poderia ser consultada na íntegra. Foram excluídos da participação na pesquisa aqueles voluntários que não trabalhavam na cidade de São Paulo e/ou que deixassem uma das questões sem resposta completa ou assinalada.

As diferenças de expressão e associações foram consideradas significantes estatisticamente quando $p<0,05$. Todas

Tabela 1 - Questões apresentadas aos pediatras participantes e as respectivas recomendações da diretriz

\begin{tabular}{|c|c|}
\hline Questões & Recomendações \\
\hline $\begin{array}{l}\text { Toda criança, a partir de qual idade, deve ter a determinação do colesterol total } \\
\text { (CT) realizada por meio de exame de sangue capilar da polpa digital? }\end{array}$ & - A partir dos 10 anos de idade. \\
\hline $\begin{array}{l}\text { As crianças e os adolescentes que apresentarem CT }>150 \mathrm{mgdL} \text { e }<170 \mathrm{mgdL} \\
\text { deverão ter qual orientação? }\end{array}$ & $\begin{array}{l}\text { - Seus pais deverão ser orientados em relação a medidas de mudança de } \\
\text { estilo de vida e o exame de CT deverá ser repetido anualmente. }\end{array}$ \\
\hline $\begin{array}{l}\text { As crianças e os adolescentes que apresentarem CT>170mgdL deverão ter qual } \\
\text { orientação? }\end{array}$ & $\begin{array}{l}\text { - } 0 \text { perfil de CT>170mgdL requer uma análise completa de lípides após } \\
\text { jejum de } 12 \text { horas. }\end{array}$ \\
\hline Crianças a partir de qual idade devem ter a aferição da pressão arterial realizada? & - A partir dos 3 anos de idade. \\
\hline Quando é considerado estado de pré-hipertensão em crianças? & $\begin{array}{l}\text { - Quando a pressão sistólica em percentis for }>90 \text { e ou quando a pressão } \\
\text { diastólica em percentis for }<95 \text { ou sempre que a pressão arterial for } \\
>120-80 \mathrm{mmHg} \text {. }\end{array}$ \\
\hline $\begin{array}{l}\text { A partir da idade mínima, com que frequência deverá ser realizada a aferição da } \\
\text { pressão arterial nas crianças? }\end{array}$ & - Em toda avaliação de saúde, pelo menos $1 x$ ao ano. \\
\hline $\begin{array}{l}\text { Segundo recomendação do National Center for Health Statistics (NCHS) quais } \\
\text { seriam as características das crianças portadoras de sobrepeso e obesidade } \\
\text { respectivamente? }\end{array}$ & $\begin{array}{l}\text { - Crianças com IMC> percentil } 95 \text { são classificadas como obesas. } \\
\text { Crianças com IMC entre os percentis } 85 \text { e } 95 \text { são consideradas como } \\
\text { portadoras de sobrepeso. }\end{array}$ \\
\hline
\end{tabular}


as análises foram realizadas usando teste de Quiquadrado e teste de Quiquadrado de tendência linear através dos Softwares Primer of Biostatistics ou SPSS for Windows.

\section{Resultados}

Um total de 370 pediatras relataram realizar atendimento pediátrico na cidade de São Paulo (SP). Participaram pediatras não especialistas (residentes) e especialistas em neonatologia, terapia intensiva, pediatria geral, cardiologia, hebiatria e endocrinologia.

Dos entrevistados, $65,7 \%$ afirmaram não ter tido conhecimento prévio da diretriz. Dentre os participantes havia 213 do sexo feminino e 157 do sexo masculino. O valor de corte utilizado para cálculo dos acertos foi $\geq 70 \%$, o que corresponde a um total de acertos $\geq$ cinco questões.

Do total de pediatras participantes, apenas $136(36,7 \%)$ alcançaram o valor de corte e dentre esses não foi encontrada diferença estatisticamente significativa $(p=0,25)$ no conhecimento da diretriz em relação aos sexos: $61,8 \%$ eram do sexo feminino apresentando $39 \%$ de acertos e $38,2 \%$ eram do sexo masculino com 33\% de acertos.

Os dados da Figura 1 mostram que dos 187 pediatras envolvidos em atividades acadêmicas, $24 \%$ acertaram o valor de corte, enquanto que de 183 pediatras não participantes de atividades acadêmicas, apenas $12,7 \%$ acertaram igual ou acima de cinco questões com diferença estatística significante $<0,001$.

A Figura 2 mostra a relação entre local de trabalho e quantidade de acertos dos pediatras participantes. Comparando o grupo que realiza atendimento em locais particulares aos demais grupos (em locais públicos e em locais públicos mais particulares) observa-se que pediatras do setor público apresentaram melhor conhecimento da prática preventiva da aterosclerose $(p=0,01)$.

A relação entre tempo de formado e quantidade de acertos e erros dos 370 pediatras de SP está destacada na Figura 3 $(p=0,07)$. Foi observada alta incidência de erros independente do tempo dedicado à pediatria. A aplicação do teste de Quiquadrado para verificação de tendência linear mostrou que não há diferença estatística significativa no aumento de erros com o passar dos anos.

Figura 1 - Distribuição dos acertos e erros (\%) dos pediatras da cidade de SP relacionada ao seu envolvimento ou não em atividades acadêmicas. Acadêmicos $n=187$ e Não Acadêmicos $n=183(p<0,001)$.

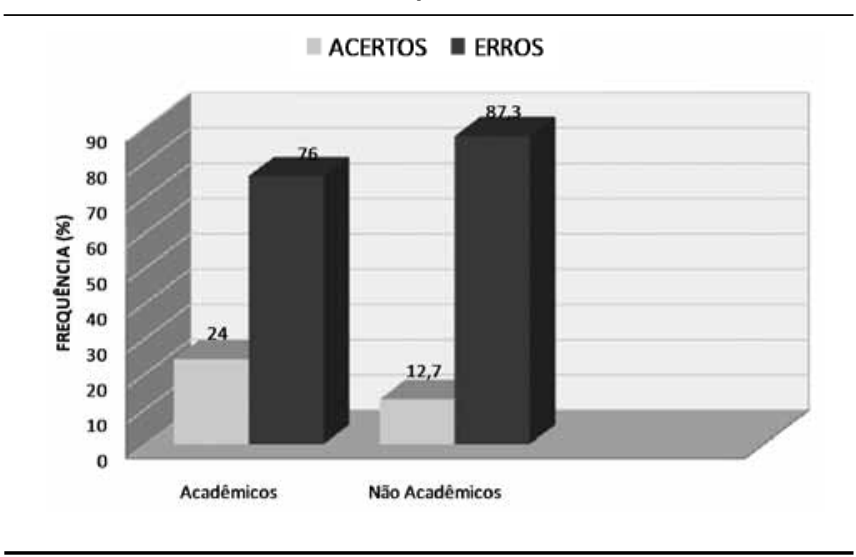

Figura 2 - Relação entre locais de trabalho (particular, público ou particular mais público) e porcentagem de acertos e erros dos pediatras de SP. Particular $n=89$; Público $n=89$ e Público+Particular $n=192(p=0,01)$

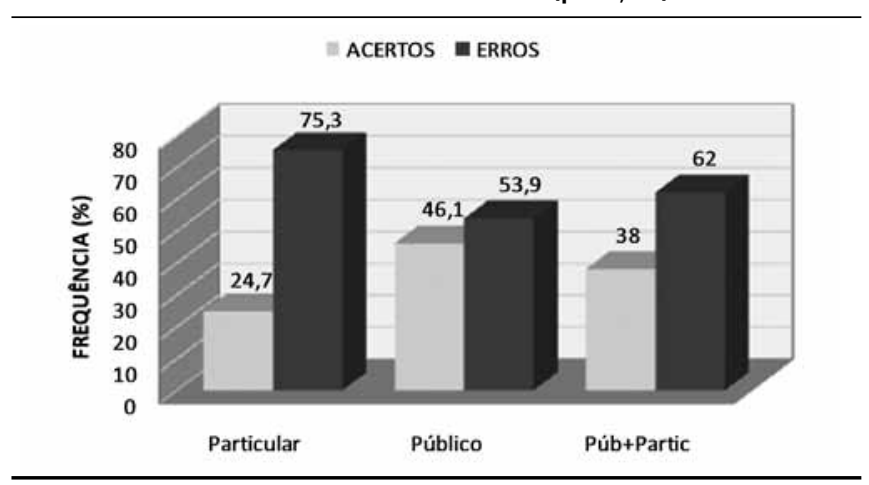

Figura 3 - Relação entre tempo de formatura (anos) e porcentagem de acertos e erros dos pediatras de SP. Intervalos de 1 a 10 $n=136,11$ a $20 n=79,21$ a $30 n=113$ e acima de $30 n=33$ $(p=0,07)+$

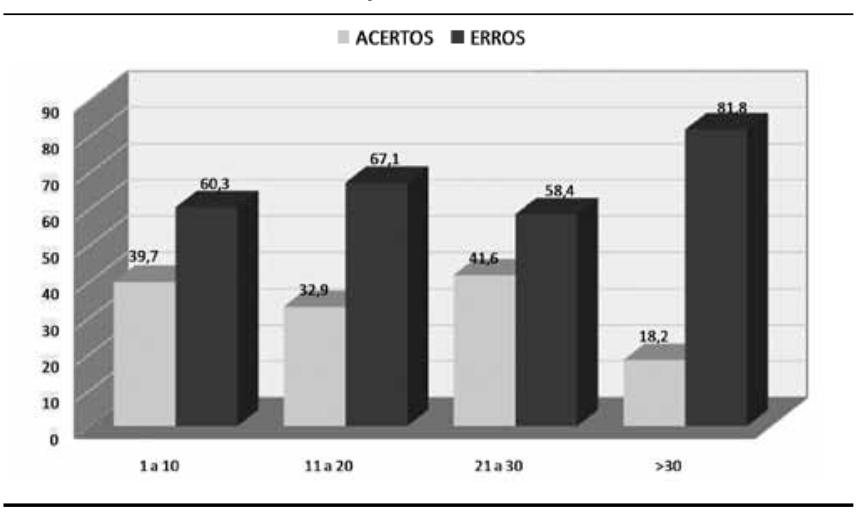

\section{Discussão}

A população estudada mostrou desconhecimento das recomendações publicadas na primeira diretriz de Prevenção da Aterosclerose na Infância e Adolescência em 2005, visto que cerca de 2/3 dos participantes afirmaram não ter tido prévio contato com a mesma e não alcançaram o valor de corte. 0 índice de questões erradas foi estatisticamente maior no grupo que declarou não participar de atividades acadêmicas. Maior porcentagem de erros também foi verificada dentre os pediatras do setor particular em relação ao setor público. Foi observada alta incidência de erros independente do tempo decorrido a partir do ano de formatura do profissional. Não houve relevância estatisticamente significativa entre tempo de formatura e conhecimento das recomendações publicadas na diretriz. Pediatras do sexo masculino não mostraram diferença estatisticamente significativa quanto ao grau de desconhecimento das respostas corretas em relação às pediatras do sexo feminino, mesmo sendo este último grupo mais numeroso na pesquisa.

A afirmação de desconhecimento da diretriz e o alto índice 
de respostas discordantes levam a uma grande preocupação com a capacidade de identificação precoce dos fatores de risco cardiovasculares e a aplicação das recomendações na prática clínica pelos pediatras da cidade de São Paulo. A população estudada se mostrou inadequadamente preparada para identificar situações clínicas oportunas e praticar a prevenção primária com aconselhamento de mudanças de estilo de vida. $\mathrm{Na}$ grande parcela da população jovem, os fatores de risco como dislipidemia, estados pré-hipertensivos e hipertensivos, tabagismo, sobrepeso e obesidade, sedentarismo e outros são, primariamente, consequências do estilo de vida da criança, do jovem ou de sua família, constituindo uma oportunidade para intervenções que podem mudar a severidade da aterosclerose na fase adulta.

Apesar das recomendações sobre o controle dos fatores de risco para a prevenção da doença coronariana serem frequentes dentre as sociedades médicas, estudos mostram grande dificuldade na aderência dos profissionais a essas recomendações tanto no Brasil como em outros países. Tal fato foi observado no grupo de pediatras entrevistados para o National Survey of Primary Care Physicians ${ }^{17}$ patrocinados pelo National Heart, Lung and Blood Institute (NHLBI), em 1988, que mostrou ser conservador quanto ao tratamento de riscos cardiovasculares, utilizando frequentemente terapia medicamentosa para níveis variáveis de colesterol sanguíneo. Somente metade dos participantes afirmou estar preparada para recomendar uma dieta redutora de colesterol e somente $14 \%$ deles sentiam-se seguros nesta recomendação. Menos de 25\% citaram três dos maiores fatores de risco para doença cardiovascular (altos valores de colesterol, hipertensão e tabagismo).

No Second NHLBI Survey of Primary Care Physicians ${ }^{18}$ já havia as orientações de uma terapia standard recomendada pelo NCEP Guidelines for Children e, mesmo assim, constatou-se uma frequência ligeiramente menor $(75,7 \%)$ de avaliações dos níveis de colesterol sanguíneo dos pacientes pediátricos comparado aos dados da primeira avaliação. A maioria (71\%) aconselhou alterações na dieta como primeiro passo para redução do colesterol, mas $16 \%$ também usaram associação de terapia medicamentosa. Da mesma forma, 25\% recomendaram drogas hipotensoras às crianças e adolescentes para tratamento da hipertensão. Nenhuma melhora notável foi verificada no reconhecimento dos fatores de risco cardiovasculares dentre os médicos entrevistados.

No Brasil são limitados os trabalhos que busquem levantar o grau de adesão de profissionais às recomendações das diretrizes $^{19,20}$. Em um deles, a amostra de 1.215 estudantes brasileiros de 7 a 17 anos de ambos os gêneros foi pesquisada quanto à frequência de indivíduos que já foram submetidos à Medida de Pressão Arterial (MPA) e o número de vezes a que foram submetidos, dentre outras informações. Apenas 28,6\% admitiram haver medido a pressão arterial previamente e 0 fizeram apenas uma vez e 53\% realizaram a MPA em menos de um ano. Houve associação entre prévia MPA com a faixa etária de 15 a 17 anos e não com idades menores. Além disso, 31\% dos participantes relatou realizar MPA em outro local que não em consultas de saúde, mostrando que, provavelmente, não resultou em nenhuma ação específica de saúde como prevenção ou terapêutica. Os 12 estudantes que foram informados estar com pressão alta na época da aferição não receberam orientação para realizar avaliação médica por esse motivo.

Uma diretriz pode requerer, em média, um período de cinco anos para ser amplamente divulgada e suas recomendações passarem a ser transpostas para a prática clínica segundo mostrou o European Action on Secondary and Primary Prevencion by Intervention to Reduce Events III (EUROASPIRE III)21. A primeira diretriz de Prevenção da Aterosclerose na Infância e Adolescência foi lançada em 2005 e está prestes a completar o seu período de divulgação, sem, no entanto, ser conhecida por muitos profissionais, pelo menos na cidade de São Paulo.

Tendo em vista o inquestionável e importante papel do pediatra na prevenção primária dos fatores de risco para a aterosclerose, os dados aqui apresentados apontam para a necessidade dos especialistas serem encorajados a identificar os fatores de risco cardiovasculares dentre os jovens, defenderem rigidamente mudanças no estilo de vida desde a mais tenra idade e, se necessário, implantar tratamento farmacológico que modificará a evolução individual desses fatores. Mudanças na saúde pública associadas às transformações sociais e culturais também são necessárias para atingir a população como um todo. A participação dos pais e médicos em interação com os demais profissionais da saúde, educadores, cientistas e legisladores é fundamental para garantir a aplicabilidade das recomendações fornecidas pela diretriz ${ }^{22,23}$.

\section{Conclusão}

A meta da diretriz, que é servir como referência para o estabelecimento de estratégias individuais e populacionais no controle dos fatores de risco para a aterosclerose desde a mais tenra idade, não foi alcançada na cidade de São Paulo, mesmo depois de transcorridos quatro anos de sua publicação. É preocupante o fato de que aliado ao não conhecimento das recomendações publicadas na primeira diretriz de Prevenção da Aterosclerose na Infância e Adolescência esteja o não cumprimento da prática diária da prevenção de transtornos metabólicos e cardiovasculares que têm início na infância e que podem se tornar doenças crônicas tardias. Sendo assim, divulgação mais adequada e cursos de educação médica continuada em que o conhecimento da diretriz se torne mais efetivo são sugeridos para corrigir tais achados.

\section{AGRADECIMENTOS}

1 - Dra Sandra J. F. E. Grisi, Dr. Alfredo E. Gilio e Sociedade de Pediatria de São Paulo (SPSP).

\section{Conflito de interesse: não há}

\section{SUMmary}

LACK OF KNOWLEDGE OF GUIDELINES FOR PREVENTION OF ATHEROSCLEROSIS IN CHILDHOOD AND ADOLESCENCE BY PEDIATRICIANS IN THE CITY of São Paulo

OBJECTIVE. To report the extent of knowledge of pediatricians in São Paulo concerning the Guidelines.

METHODS. At pediatric events or hospitals, pediatricians were given a questionnaire about the time of training, work, dedication in the area of pediatrics, and academic performance for reply to 
multiple choice questions with information drawn from the Guidelines. The Chi -square and the Chi -square of linear trend tests were chosen for statistical calculations.

REsults. Among 370 pediatricians surveyed, 65.7\% had no previous knowledge of the Guidelines. The cut-off for the hits was $>$ or $=70 \%$ (> or $=5$ correct replies). Only 136 pediatricians (36.7\%) reached the cut-off and no gender differences were found in knowledge ( $p=0.25$ ). Among 187 professionals involved in academic activities, 45 (24\%) hit the cut-off and about 183 participants from non-academic activities, 23 (12.7\%) reached $>$ or $=$ 5 hit points $(p<0.001)$. Pediatricians in the public sector had better knowledge about preventive measures for atherosclerosis risk factors (46.1\%, $p=0.01$ ). Awareness of the Guidelines was independent from training time.

CONCLUSION. The majority of pediatricians in the city of São Paulo were not familiar with the Guidelines for Prevention of Atherosclerosis in Childhood and Adolescence and individual strategies were rarely found. Medical education and adequade disclosure of the Guidelines are necessary for active control of populational risk factors. [Rev Assoc Med Bras 2010; 56(2): 157-61]

KEY WORDS: Health planning guidelines. Primary prevention. Atherosclerosis. Timeout expired. The timeout period elapsed prior to completion of the operation or the server is not responding. Adolescent.

\section{REFERÊNCIAS}

1. National Cholesterol Education program (NCEP). Highlights of the report of the expert Panel on Blood Cholesterol Levels in Children and Adolescents. Pediatrics. 1992;89:495-501

2. Hayman LL, Meininger JC, Daniels SR, McCrindle BW, Helden L, Ross J, et al. American Heart Association Committee on Atherosclerosis, Hypertension, and Obesity in Youth of the Council on Cardiovascular Disease in the Young; American Heart Association Council on Cardiovascular Nursing; American Heart Association Council on Epidemiology and Prevention; American Heart Association Council on Nutrition, Physical Activity, and Metabolism. Primary prevention of cardiovascular disease in nursing practice: focus on children and youth: a scientific statement from the American Heart Association Committee on Atherosclerosis, Hypertension, and Obesity in Youth of the Council on Cardiovascular Disease in the Young, Council on Cardiovascular Nursing, Council on Epidemiology and Prevention, and Council on Nutrition, Physical Activity, and Metabolism. Circulation. 2007;116(3):344-57.

3. Gianinni SD. História natural da aterosclerose. Rev Soc Cardiol Estado de São Paulo. 2000;10(6):677-85

4. Françoso LA, Coates V. Evidências Anatomopatológicas do início da aterosclerose na infância e adolescência. Arq Bras Cardiol. 2002;78:131-6.

5. Newman III WP, et al. Relation of serum lipoprotein levels and systolic blood pressure to early atherosclerosis: the Bogalusa Heart Study. N Engl J Med. 1986; 314: 138-44.

6. Berenson GS, Wattigney WA, Tracy RE, Newman WP 3rd, Srinivasan SR, Webber LS, Tracy RE, Newman WP 3rd, Herderick EE, et al. Atherosclerosis of the aorta and coronary arteries and cardiovascular risk factors in person aged 6 to 60 years and studied at necropsy (The Bogalusa Heart Study). Am J Cardiol. 1992;70:851-8.
7. Tracy RE, Newman WP, Wattigney WA, Berenson GS. Risk factors and atherosclerosis in youth: autopsy findings of The Bogalusa Heart Study. Am J Med Sci. 1995; 310(Suppl 1):S37-S41.

8. The Pathobiological Determinants of the Atherosclerosis in Youth (PDAY) Research Group. Natural history of aortic and coronary atherosclerosis lesions in youth: findings from the PDAY Study. Arterioscler Thromb. 1993;13: 1291-8.

9. Strong JP, Malcom GT, McMahan CA, et al. Prevalence and extent of atherosclerosis in adolescents and young adults: implications for prevention from the Pathobiological Determinants of Atherosclerosis in Young Study. JAMA. 1999; 281:727-35.

10. Davis PH, Dawson JD, Riley WA, Lauer RM. Carotid intimal-medial thickeness is related to cardiovascular risk factors measured from childhood through middle age: the Muscatine Study. Circulation. 2001;104:2815-9.

11. Li S, Chen W, Srinivasan SR, et al. Childhood cardiovascular risk factor and carotid vascular changes in adulthood: the Bogalusa Heart Study. JAMA. 2003;290:2271-6.

12. Raitakari OT, Juonala M, Kahonen M, Taittonen L, Laitinen T, Mäki-Torkko $\mathrm{N}$, et al. Cardiovascular risk factors in childhood and carotid intima-media thickness in adulthood: the Cardiovascular Risk in Young Finns Study. JAMA. 2003;290:2277-83.

13. Giuliano IC, Coutinho MS, Freitas SF, Pires MM, Zunino JR, Ribeiro PQ. Lípides séricos em crianças e adolescentes da rede escolar de Florianópolis - Estudo Floripa Saudável 2004. Arq Bras Cardiol. 2005;85:85-91.

14. Gama SR, Carvalho MS, Chaves CRMM. Prevalência em crianças de fatores de risco para as doenças cardiovasculares. Cad Saúde Pública. 2007;23:2239-45.

15. Pellanda LC, Echenique L, Barcellos LMA, Maccari J, Borges FK, Zen BL. Doença cardíaca isquêmica: a prevenção inicia durante a infância. J Pediatria (Rio de J). 2002;78:91-6.

16. Giuliano ICB, Caramelli B, Pellanda L, Duncan B, Mattos S, Fonseca FAH, editores. I Diretriz de Prevenção da Aterosclerose na Infância e na Adolescência. Arq Bras Cardiol. 2005;85:3-36.

17. Kimm SY, Payne GH, Lakatos E, Darby C, Sparrow A. Management of cardiovascular disease risk factors in children. A national survey of primary care physicians. Am J Dis Child. 1990;144:967-72.

18. Kimm SY, Payne GH, Stylianou MP, Waclawiw M, Lichtenstein C. National trends in the management of cardiovascular disease risk factors in children: second NHLBI Survey of Primary Care Physicians. Pediatrics. 1998;102:1-8.

19. Santos RD, Spósito AC, Santos JE, Fonseca FH, Moriguchi EH, Martinez TL, et al. Programa de Avaliação Nacional do Conhecimento sobre Prevenção da Aterosclerose (PANDORA). Um questionário entre cardiologistas brasileiros sobre redução do colesterol. Arq Bras Cardiol. 2000; 75:289-95.

20. Da Silva MAM, Rivera IR, Souza MGB, Carvalho ACC. Medida da pressão arterial em crianças e adolescentes: recomendações das diretrizes de hipertensão arterial e prática médica Atual. Arq Bras Cardiol. 2007;88:491-5.

21. Kotseva K, Wood D, De Backer G, De Bacquer D, Pyorala K, Keil U on behalf of the EUROASPIRE Study group. EUROASPIRE III: a survey on the lifestyle, risk factors and use of cardioprotective drug therapies in coronary patients from 22 European countries. Eur J Cardiol Prev Rehab. 2009;16:121-37.

22. McGill HC, McMahan CA, Gidding SS. Are pediatricians responsible for prevention of adult cardiovascular disease? Clinical Advance. Nat Clin Pract Cardiovasc Med. 2009;6:10-1.

23. McGill HC Jr, McMahan CA, Gidding SS. Preventing heart disease in the $21^{\text {st }}$ Century: implications of the Pathobiological Determinants of Atherosclerosis in Youth (PDAY) Study. Circulation. 2008;117:1216-27.

Artigo recebido: 15/08/09 Aceito para publicação: 24/11/09 